\title{
Increased Sensitivity of Platelets from Unstable Angina Patients to Adenosine Diphosphate-Induced Aggregation
}

\author{
Gomathy VISWANATHAN ${ }^{1, *}$ and P.T.Viswambharan NAIR ${ }^{2}$ \\ ${ }^{1}$ Radiation Biology and Biochemistry Division and \\ ${ }^{2}$ Medical Division, Bhabha Atomic Research Centre, \\ Bombay 400 085, India
}

(Received November 22, 1993)

\begin{abstract}
Summary ADP-induced platelet aggregation of normal individuals and of patients with unstable angina were studied. Abnormalities of platelet function included increased aggregability. The ADP concentration required for half-maximal aggregation was significantly lower $(4.28 \pm 0.30 \mu \mathrm{M})$ in the unstable angina patients compared with that for normal individuals $(5.24 \pm 0.15 \mu \mathrm{M})$. The results are suggestive of platelet aggregation as a useful prognostic indicator for unstable angina.
\end{abstract}

Key Words: platelet aggregation, unstable angina

Platelets have an important role in the initiation and maintenance of arteriosclerosis. Their role in the pathogenesis of unstable angina and myocardial infarction has also been documented [1-5]. Increased biosynthesis of eicosanoids was observed in patients with unstable angina in contrast to their levels of synthesis in normal individuals and in patients with stable coronary disease both at rest and in exercise-induced myocardial ischemia, suggesting episodic platelet activation leading to coronary occlusion either by formation of occlusive thrombi or by release of vasoactive compounds by platelets [6]. Measuring aggregation in vitro using whole blood aggregometry in response to ADP can provide rapid diagnosis of in vivo platelet abnormalities [7]. Significant increases in ADP-induced platelet aggregation has been found in diabetics [8]. Similar increase associated with thromboxane formation has been shown after acute ethanol ingestion [9]. The present study reports our findings on ADP-induced aggregation of platelets in patients admitted to our intensive care unit with unstable angina.

*To whom correspondence should be addressed. 


\section{MATERIALS AND METHODS}

Subjects. The study group consisted of 47 normal healthy subjects and 27 patients. Unstable angina was defined as chest pain occurring at rest for a minimum period of $10-15 \mathrm{~min}$ on more than one occasion in the absence of increased heterodimeric (muscle-MM, brain-BB isoenzyme) fraction of creatine kinase in patients with evidence of coronary artery disease based on electrocardiographic changes indicating ischemia during episodes of chest pain. Control subjects were healthy normal individuals. Individuals who had consumed aspirin or other analgesics were excluded from both groups.

Platelet aggregation. Five milliliters of blood was collected in $0.5 \mathrm{ml}$ of $3.8 \%$ sodium citrate diluted 1:2 with saline, and 1-ml aliquots were dispensed in cuvettes containing Teflon-coated stir bars. Aggregation was carried out by the impedance method [10] between one-half and two hours of sampling in a Chronolog whole blood aggregometer (Chronolog Corp., Havertown, PA) with ADP (Sigma Chemical Co., St. Louis, MO) used as the agonist. Nine different concentrations of ADP $(2-10 \mu \mathrm{M})$ were added, and the change in impedance was recorded as a function of time on a strip chart recorder. Rate of aggregation was evaluated by the slopes of the first wave of the aggregation curves [11]. The slopes for different ADP concentrations were plotted, and the ADP concentration inducing half-maximal aggregation was determined graphically. This value was used as the parameter for comparison [12].

Lipids. Total cholesterol and triglycerides were measured enzymatically by use of standard diagnostic kits (A. Menarini Diagnostics, Florence, Italy).

\section{RESULTS}

The clinical and biochemical data of normal and unstable angina patients are tabulated in Table 1. No significant difference in the mean age or blood pressure was seen between the two groups. However, there was a significant difference in the plasma mean total cholesterol (TC, $p<0.001)$ and triglyceride $(\mathrm{TG}, p<0.05)$ levels.

Platelet aggregation expressed as that concentration of ADP $(\mu \mathrm{M})$ required for half-maximal aggregation is also shown in Table 1. A significant increase in the mean platelet aggregability of unstable angina patients was observed $(p<0.01)$ compared with that of the normals, as seen by a decrease in the ADP concentration needed for half-maximal aggregation. As seen from the histogram shown in Fig. 1, platelets from 9 out of 27 patients aggregated at concentrations between 2 and 3.5 $\mu \mathrm{M}$ ADP, while none from the control group aggregated at such low concentrations. With increasing concentration of ADP there was a decline in the number of angina patients aggregating in the respective group. On the other hand, the control group showed an increase in the ADP concentration required for half-maximal 
Table 1. Characteristics of the study population.

\begin{tabular}{lcc}
\hline & Unstable angina & Normal \\
\hline Age (years) & $56.4 \pm 2.24$ & $44.2 \pm 8.75$ \\
Sex (female/male) & $5 / 22$ & $19 / 28$ \\
Blood pressure $(\mathrm{mmHg})$ & $123.5 \pm 2.4$ & $125 \pm 2.2$ \\
& $82 \pm 1.8$ & $81 \pm 2.1$ \\
Total cholesterol $(\mathrm{mg} / \mathrm{dl})$ & $208.94 \pm 9.05^{* * *}$ & $165.83 \pm 4.04$ \\
Triglycerides $(\mathrm{mg} / \mathrm{dl})$ & $153.94 \pm 14.09^{*}$ & $114.3 \pm 5.9$ \\
ECG & ST-T changes & - \\
& suggestive of coronary \\
Platelet aggregation ${ }^{\mathrm{a}}(\mu \mathrm{M}$ ADP) & heart disease & $5.24 \pm 0.15$ \\
\hline Values are expressed as means $\pm \mathrm{SD} .{ }^{a}$ Expressed as concentration of ADP required for \\
half-maximal aggregation. ${ }^{*} p<0.05,{ }^{* *} p<0.01,{ }^{* * *} p<0.001$ vs. corresponding normal \\
$\quad$ value.
\end{tabular}

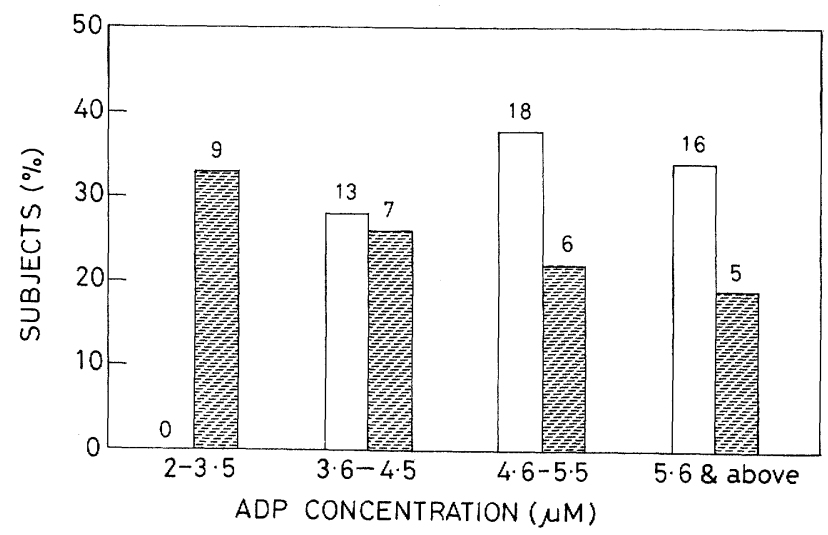

Fig. 1. Percentage distribution of individuals against ADP concentration required for

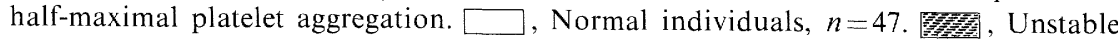
angina patients, $n=27$.

aggregation in 34 out of 47 individuals whose platelets aggregated at concentrations above $4.6 \mu \mathrm{M}$.

\section{DISCUSSION}

Thromboxane formation is known to be increased several fold during platelet activation and is therefore an important pathomechanism with diagnostic and therapeutic potential. However, analysis of in vivo thromboxane $\mathrm{A}_{2}$ formation is complicated by the fact that it can be inactivated nonenzymatically to thromboxane $B_{2}$, which is then metabolized to products that are also natural metabolites. Although errors due to sampling and analysis can be avoided, analysis of thromboxane and prostacyclin would be time consuming for undertaking early antiVol. 16, No. 3, 1994 
thrombotic therapeutic intervention. Using a whole blood platelet aggregometer, we have shown that $33 \%$ of unstable angina patients have increased platelet aggregability requiring less than $3.6 \mu \mathrm{M}$ ADP for half-maximal aggregation, whereas $72 \%$ of normal individuals required $4.6 \mu \mathrm{M}$ ADP and above. Platelet aggregation could therefore be used as an independent biochemical criterion for optimizing therapeutic intervention.

The response of patients with symptoms of unstable angina and acute coronary insufficiency to antiplatelet drugs such as aspirin is well documented. Many cases of unstable angina lead to myocardial infarction. Impending plaque rupture, thrombus formation, and subsequent coronary occlusions are the hallmark of acute myocardial infarction. Evaluation of platelet aggregation could therefore provide the basis for an effective antithrombotic approach.

The authors wish to acknowledge Dr. J.M. Noronha, Dr. B.B. Singh, and Dr. U.A. Desai for their valuable advice and encouragement.

\section{REFERENCES}

1. Fuster, V., Steele, P.M., and Chesebro, J.H. (1985): Role of platelets and thrombosis in coronary atherosclerotic disease and sudden death. J. Am. Coll. Cardiol., 5, 175B-181B.

2. Haft, J.T. (1979): Role of platelets in coronary artery disease. Am. J. Cardiol., 43, $1197-$ 1206.

3. Schafer, A.I., and Handin, R.I. (1979): The role of platelets in thrombotic and vascular disease. Prog. Cardiovasc. Dis., 22, 31-52.

4. Falk, E. (1985): Unstable angina with fatal outcome, dynamic coronary thrombosis leading to infarction and/or sudden death, autopsy evidence of recurrent mural thrombosis with peripheral embolization culminating in total vascular occlusion. Circulation, 71, 699-708.

5. Davies, M.J., Thomas, A.C., Knapman, P.A., and Hangartner, J.R. (1986): Intramyocardial platelet aggregation in patients with unstable angina suffering sudden ischaemic cardiac death. Circulation, 73, 418-427.

6. Fitzgerald, D.J., Louis Roy, M.B., Catella, F., and Fitzgerald, G.A. (1986): Platelet activation in unstable angina. N. Engl. J. Med., 315, 983-989.

7. Zawilska, K.M., Born, G.V.R., and Begent, N.A. (1982): Effect of ADP-utilizing enzymes on arterial bleeding time in rats and rabbits. Br. J. Haematol., 50, 317-325.

8. Jethmalani, S.M., Viswanathan, G., and Noronha, J.M. (1989): Relationships among plasma lipids, lipoproteins, and platelet aggregation profiles in diabetes. J. Clin. Biochem. Nutr., 6, 257-264.

9. Hilbom, M., Kangasaho, M., Kaste, M., Numminen, H., and Vapaatalo, H. (1985): Acute ethanol ingestion increases platelet reactivity: Is there a relationship to stroke? Stroke, 16, 19-23.

10. Cardinal, D.C., and Flower, R.J. (1980): The electronic aggregometer: A novel device for assessing platelet behaviour in blood. J. Pharmacol. Methods, 3, 135-138.

11. Gaxiola, B., Friedl, W., and Propping, P. (1984): Epinephrine-induced platelet aggregation. A twin study. Clin. Genet., 26, 543-548.

12. Jethmalani, S.M., Viswanathan, G., and Noronha, J.M. (1989): Relationships between plasma lipids, lipoproteins and platelet aggregation profiles in essential hypertension. $J$. Clin. Biochem. Nutr., 6, 245-255. 